\title{
Evaluation of ONU Power Saving Modes in Next Generation Optical Access Networks
}

\author{
Abhishek Dixit, Bart Lannoo, Sofie Lambert, Didier Colle, Mario Pickavet, Piet Demeester \\ Department of Information Technology, Ghent University - IBBT, Gent, Belgium, \\ abhishek.dixit@intec.ugent.be
}

\begin{abstract}
We propose a new dynamic bandwidth allocation algorithm for energy efficiency in next generation optical access (NGOA) networks, and evaluate the power savings possible at the optical network unit (ONU) by applying sleep and doze modes. Sleep mode is found to be most effective for NGOA systems with burst mode traffic transmission and reception.
\end{abstract}

\section{Introduction}

There is a large effort to reduce energy consumption for minimizing network operating expenses, environmental impacts, and carbon footprints besides meeting tougher regulations. Next to data centers, the access network consumes a major part of the total energy consumption and the equipment power consumption is the dominant contributing factor to the total carbon footprint ${ }^{1}$. Optical access networks are inherently more energy efficient than their previous counterparts (e.g. ADSL and VDSL) and the optical network unit (ONU), installed at the customer premises, accounts for the major part of the energy consumed. We evaluate ONU power consumption in various next generation optical access (NGOA) architectures and the possible savings from sleep and doze modes. In our knowledge, this is the first paper which studies the effect of power saving modes in various NGOA architectures.

\section{Optical Access Technologies}

NGOA systems are actively considered for higher data rates, long reach, high customer fan out and energy efficiency ${ }^{2}$. To meet NGOA requirements, different system concepts are proposed such as high bit rate time division multiple access (HB-TDMA) passive optical networks (PON), wavelength division multiplexing (WDM) PONs, hybrid TDMA/WDM PONs (TWDM PON), point-to-point (PtP) and active optical networks (AON).

HB-TDMA-PON: High bit rate (40 Gbps) TDMA-PONs are based on non-return to zero (NRZ) on-off keying. The advantages of HBTDMA-PON are its easy migration capabilities and high flexibility in resource allocation. However, it suffers from reach limitations posed by serious dispersion issues with 40G NRZ transmission. Hence, special functionalities like dispersion compensation and optical power amplification are required.
WDM-PON: WDM-PON offers the most straightforward way of capacity increase. It minimizes the complexities needed for TDMA and has a potentially high reach and security. However, migration, low customer fan out, and static resource allocation are some of the major issues with WDM-PON. We consider two variants of WDM-PON: with tunable lasers (WDM-TL) and with reflective semiconductor optical amplifiers (WDM-RSOA) at the ONU.

PtP: In PtP, each ONU is connected directly via a fiber to the central office (CO). It requires a large duct space and footprint at the CO.

$A O N$ : AONs use an active remote node in the field that requires powering and maintenance. However, it increases flexibility.

TWDM-PON: TWDM-PON combines the flexibility of TDMA-PON with the increased overall capacity of WDM technology. We consider two variants: split (S-TWDM) and switched (Sw-TWDM) ${ }^{4}$.

Besides, the power consumption in gigabit passive optical network (GPON) is analyzed to benchmark the results.

\section{ONU power consumption model}

In Fig. 1, we present the power consumption model of the ONU. The model is motivated by ${ }^{1-3}$ and a large survey of component datasheets. The ONU may support gigabit Ethernet (GbE), digital optics (DO), analog optics and multimedia over co-axial alliance (MoCA) capabilities. However, we consider the support of only basic functionalities like GbE and DO. The miscellaneous and power conversion losses are assumed at $5 \%$ and $20 \%$, respectively. The memory requirement in different concepts may vary, and we have assumed a power consumption of $30 \mathrm{~mW}$ per MB of memory. The system on chip (SoC) and Dual subscriber line interface circuit (SLIC) power consumptions are assumed for a simple point to point link with no additional functionalities. For a specific system, 


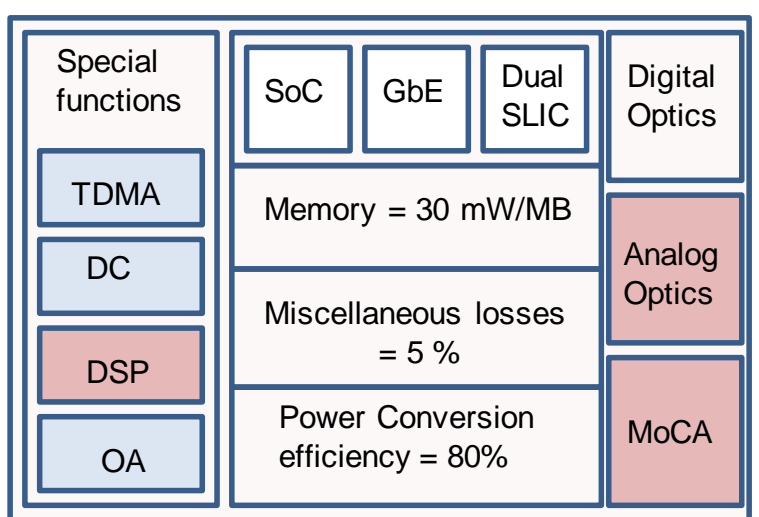

Fig. 1: ONU power consumption model. Red blocks show the sub-components that are not considered, blue blocks show the sub-components that are only considered for NGOA systems that require them.

we add the power consumption for special functionalities like TDMA, dispersion compensation (DC), optical amplification (OA), and digital signal processing (DSP), as required. For example, we add TDMA power consumption for TWDM-PON and GPON, and TDMA, DC and OA functionality for HB-TDMA-PON. DSP is required for system concepts like orthogonal frequency division multiplexing (OFDM) PON which is not considered in this paper.

Doze and sleep modes were investigated to bring down the power consumption at the $\mathrm{ONU}^{2}$. In doze mode, non-essential functions are powered off with an additional powering off of the ONU transmitter for substantial periods of time while the receiver remains on. In sleep mode, both the ONU transmitter and ONU receiver are turned off for substantial periods of time. Tab. 1 gives the detailed power consumption of various components for active, doze and sleep mode.

\section{Sleep Awareness Algorithm}

Several mechanisms to facilitate sleep mode like physical layer operations and maintenance (PLOAM), ONT/ONU management and control interface $(\mathrm{OMCl})$ and implicit signaling are proposed $^{3}$. We use PLOAM based signaling. Furthermore, interleaved polling with adaptive cycle time (IPACT $)^{5}$ is considered as the dynamic bandwidth allocation (DBA) algorithm for scheduling upstream transmission in TDMA PON (particularly Ethernet PON). However, IPACT does not support sleep mode and thus we modified IPACT and propose a new DBA algorithm, which we refer to as the IPACT with Sleep Mode Awareness (IPACT-SMA), to evaluate sleep mode gains in the various NGOA technologies. In IPACT, downstream traffic for an ONU is transmitted on a first come first serve basis, and thus the ONU receiver has to be on at all times. First of all, we eliminate the necessity of an ONU to be awake all the time
Tab. 1: Power consumption values

\begin{tabular}{|lccc|}
\hline Components & $\begin{array}{c}\text { Active } \\
(\mathrm{mW})\end{array}$ & $\begin{array}{c}\text { Doze } \\
(\mathrm{mW})\end{array}$ & $\begin{array}{c}\text { Sleep } \\
(\mathrm{mW})\end{array}$ \\
SoC & 800 & 535 & 370 \\
Dual SLIC & 175 & 107 & 74 \\
GbE & 700 & 0 & 0 \\
TDMA & 500 & 500 & 0 \\
Optical Amplification & 2000 & 2000 & 0 \\
Dispersion Compensators & 2000 & 2000 & 0 \\
Digital Signal Processing & 4000 & 4000 & 0 \\
1 G Fixed TRX (for & & & \\
GPON/AON/PtP) & 500 & 250 & 0 \\
1 G Tunable TRX with APD (for & & & \\
WDM PON) & 1700 & 900 & 0 \\
1 G Reflective SOA based TRX & 1300 & 900 & 0 \\
with APD (for WDM-RSOA) & & & \\
10/5 G Tunable TRX with APD & & & \\
(for TWDM PON) & 2500 & 1670 & 0 \\
40/10 G TRX (for HB-TDMA & & & \\
PON) & 4000 & 3200 & 0 \\
\hline
\end{tabular}

and use PLOAM based signaling to inform an ONU about the awake period. The downstream traffic is transmitted during this awake period. This necessitates buffering for both the downstream and upstream traffic. Note that buffering introduces delay in traffic which has to be within the quality of service (QoS) limits. Further, we maximize the alignment of the transmission of upstream traffic and reception of downstream traffic for an ONU, maximizing the time during which an ONU can sleep. The optical line terminal (OLT) determines the sleep period according to the upstream and downstream bandwidth backlog of an ONU and grants a transmission slot (TS) according to:

$$
T S=\operatorname{Min}\left\{\frac{T_{\text {cycle }}}{N_{u}}, \operatorname{Max}\left(\frac{B_{u}}{R_{u}}, \frac{B_{D}}{R_{D}}\right)\right\}
$$

where Min/Max represents the minimum and maximum value of the function, $\mathrm{T}_{\text {cycle }}$ is the cycle time in which ONUs are polled, $N_{u}$ is the number of users, $B_{u}$ and $B_{D}$ are the backlogged upstream and downstream bytes for an ONU, $R_{u}$ and $R_{D}$ are the upstream and downstream data rate, respectively. Note that the ONU may be allocated a longer transmission slot than it requested, in which the newly arrived packets between the time of a previous request and present grant are transmitted. If however, the ONU has no additional packet arrivals, it goes to doze mode. The ONU sleeps for a period of $\mathrm{T}_{\text {cycle }}$ - TS. The proposed algorithm is adopted to study the sleep mode in all NGOA architectures. For example, for WDM PON, PtP, and AON, $\mathrm{N}_{\mathrm{u}}$ is chosen as 1. Furthermore, as upstream and downstream capacities are shared for WDMRSOA PON, the transmission slot is adapted to:

$$
T S=\operatorname{Min}\left\{T_{c y c l e}, \frac{B_{u}}{R_{u}}+\frac{B_{D}}{R_{D}}\right\}
$$

\section{Simulation Results}

In OPNET, we simulate various NGOA architectures, with a cycle time of $5 \mathrm{~ms}$, an overhead time for clock recovery $\left(\mathrm{T}_{\mathrm{o}}\right)$ of $125 \mu \mathrm{s}$, 
an upstream and downstream average user rate of $500 \mathrm{Mbps}$ and a peak user rate of $1 \mathrm{Gbps}$. The latter value is chosen as it is one of the key requirements of NGOA architectures ${ }^{2}$. Traffic generation parameters are chosen as in $^{5}$. Different NGOA architectures have different advantages and are proposed for different reach and fan out scenarios. As we are evaluating only the ONU power consumption, the number of users does not impact our study. However, what matters is the upstream and downstream line rate of the architectures as it is one of the main factors influencing sleep and doze periods.

Tab. 2 gives the power consumption in sleep (S), doze (D) and active (A) state and the considered downstream and upstream line rate of the architectures. The burst factor (BF) is the ratio between the upstream line rate and the average user rate, and the asymmetricity factor $(\mathrm{AF})$ is the ratio between the downstream and upstream line rate. WDM-RSOA has a higher sleep state power consumption compared to other NGOA concepts, as it requires a higher buffer (27 MB compared 0.8 MB for other NGOA systems) due to its less effective upstream and downstream line rate, resulting in a higher network load. For other concepts, sleep state power consumption is the same as they support the same essential functionalities in sleep state.

Fig. 2 gives the variation of the sleep period with BF and AF. An ONU can sleep for more time with a higher BF. Also AF affects the sleep period as with a high downstream line rate, the reception time of downstream traffic is smaller, resulting in a better alignment of upstream and downstream transmission slots. Fig. 3 shows the average and maximum variation of the queue size with the cycle time for $\mathrm{BF}=2, \mathrm{AF}=1$ and for a load of 0.5 . Fig. 4 gives the power consumption of the different NGOA architectures applying doze and sleep modes. In doze mode, an ONU remains in doze state for a period $\left(\mathrm{T}_{\mathrm{D}}\right)$ of $\mathrm{T}_{\text {cycle }}-\frac{\mathrm{T}_{\text {cycle }}}{\mathrm{BF}}$. In sleep mode, an ONU remains in sleep state for a period $\left(T_{S}\right)$ that is obtained in Fig. 2. Note that, with sleep mode, an ONU remains in doze state for a period of $\mathrm{T}_{\mathrm{D}}-\mathrm{T}_{\mathrm{S}}-\mathrm{T}_{\mathrm{O}}$. It is clear that the NGOA concepts

Tab. 2: Power consumption in various states and upstream/downstream line rates

\begin{tabular}{|c|c|c|c|c|c|}
\hline \multirow[t]{2}{*}{ System } & \multirow{2}{*}{$\begin{array}{l}\text { US/DS } \\
\text { (Gbps) }\end{array}$} & \multirow{2}{*}{$\begin{array}{l}\mathrm{BF} / \\
\mathrm{AF}\end{array}$} & \multicolumn{3}{|c|}{ Power (W) } \\
\hline & & & $A$ & $\mathrm{D}$ & $\mathrm{S}$ \\
\hline WDM-RSOA & $1 / 1$ & $1 / 1$ & 4.7 & 2.7 & 1.5 \\
\hline GPON & $1 / 1$ & $2 / 1$ & 3.4 & 1.8 & 0.6 \\
\hline WDM-TL & $1 / 1$ & $2 / 1$ & 4.3 & 1.9 & 0.6 \\
\hline PtP & $1 / 1$ & $2 / 1$ & 2.8 & 1.3 & 0.6 \\
\hline $\mathrm{AON}$ & $1 / 10$ & $2 / 10$ & 2.8 & 1.3 & 0.6 \\
\hline S-TWDM & $5 / 10$ & $10 / 2$ & 5.9 & 3.2 & 0.6 \\
\hline Sw-TWDM & $5 / 10$ & $10 / 2$ & 5.9 & 3.2 & 0.6 \\
\hline HB-TDMA & $10 / 40$ & $20 / 4$ & 9.7 & 6.2 & 0.6 \\
\hline
\end{tabular}

with a higher BF gain more with sleep mode.

\section{Conclusions}

Sleep mode is found to save about $90 \%$ when it is applied to NGOA system concepts with a burst mode transmission and reception like high bit rate TDMA-PONs and about $80 \%$ in hybrid TWDM-PONs. Moderate savings between 35 to $41 \%$ are found for WDM-PON, AON and PtP systems. With increase in polling time of users, the need of buffering increases linearly.

\section{Acknowledgements}

The research leading to these results has received funding from the European Community's Seventh Framework Programme (FP7/2007-2013) under grant agreement $n^{\circ}$ 249025 (ICT- OASE).

\section{References}

[1] B. Skubic et al., IEEE Commag. 50, 1 (2012).

[2] FP7 ICT-OASE, D4.2.1 (2011).

[3] ITU-T Rec. Series G Supplement 45, (2009).

[4] A.Dixit et al., Proc. of IEEE ANTS (2011).

[5] G. Kramer et al., IEEE Commag. 40, 2 (2002).

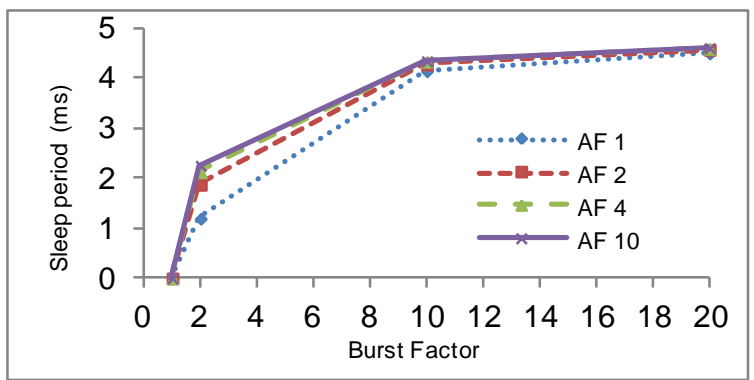

Fig. 2: Sleep period as a function of the burst factor (BF) and asymmetricity factor (AF)

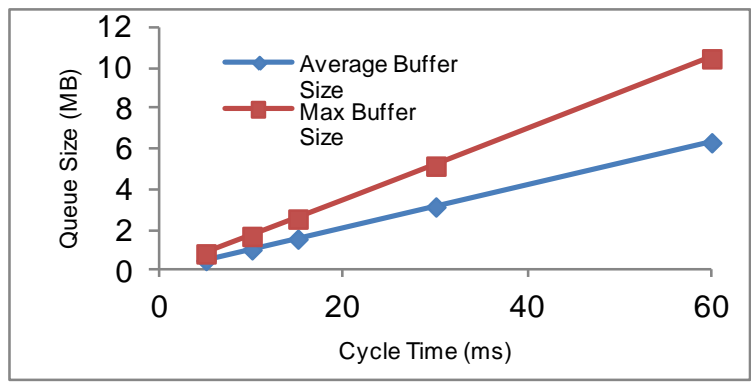

Fig. 3: Queue size as a function of the cycle time

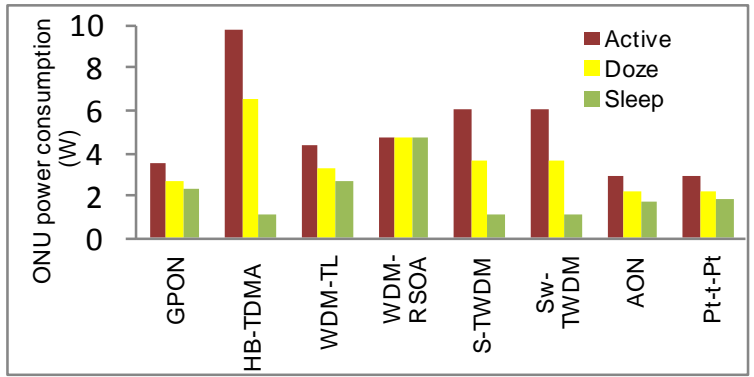

Fig. 4: ONU power consumption of various next generation optical access technologies 